\title{
Analgesia of Patients with Multiple Rib Fractures in Critical Care: A Survey of Healthcare Professionals in the UK
}

\author{
Laura Beard ${ }^{1}$, Billy Holt ${ }^{2}$, Catherine Snelson ${ }^{3}$, Chetan Parcha $^{4}$, Fang Gao Smith ${ }^{5}$, Tonny Veenith ${ }^{6}$
}

\begin{abstract}
Introduction: Good analgesia has been shown to reduce the risk of pneumonia, chronic pain, and mortality in patients with multiple rib fractures (MRFs). This survey explores the current analgesic practice in the UK, protocol use, barriers to provision, and physician preferences.

Materials and methods: A web-based survey was distributed nationally to an enriched cohort of clinicians working in UK trauma units with an interest in MRF management.

Results: Seventy-nine healthcare professionals responded. A third (31.4\%) reported that their department had a rib fracture pain protocol, $52.9 \%$ did not, and $15.7 \%$ were unsure. Significantly more respondents reported adequate pain control when a hospital protocol was present compared to when not $\left(x^{2}, p<0.01\right)$. Inadequate analgesia, a poor cough, and inability to breathe deeply were the commonest complications reported by $81.4,78.6$, and $65.7 \%$, respectively. Patient-controlled analgesia (PCA) was the most commonly used form of analgesia (38.6\%) followed by thoracic epidural (TEA) (30.0\%) and continuous opioid infusion (18.6\%). However, TEA was the preferred method of analgesia among respondents (37.1\%) followed by serratus block (21.4\%), paravertebral block (17.1\%), and PCA (14.3\%).

Discussion: There is considerable variation among physicians in their current use of analgesic modalities, with opiate-based methods predominating despite a physician preference for regional techniques. Thoracic epidurals are preferred by physicians but of limited use as a result of contraindications, time pressures, and staff skill mix. Pain control is reported to be better handled when protocols are present. Further research focusing on currently utilized regional techniques is required in order to produce a validated standardized national protocol that is informed by the current practice, the evidence base, and limitations to service provision.

Keywords: Analgesia, Critical care, Pain relief, Rib fractures, Thorax trauma.

Key messages: There is considerable variation among physicians in their current use of analgesic modalities. Opiate-based methods dominate for thoracic trauma despite a physician preference for regional techniques, which can be challenging in this cohort due to contraindications, staff skill mix, and time pressures. Inadequate analgesia is common but is better managed when pain management protocols are available. Indian Journal of Critical Care Medicine (2020): 10.5005/jp-journals-10071-23375
\end{abstract}

\section{INTRODUCTION}

Multiple rib fractures (MRFs) are commonly associated with blunt trauma present in approximately $10 \%$ of those with all types of trauma and up to $80 \%$ of those with significant thoracic injury. ${ }^{1,2}$ These patients form a high-risk cohort with a mortality as high as $33 \%$. The risk of mortality doubles in those above 65 years of age. ${ }^{3,4}$ Complications include pneumonia, pulmonary effusion, acute respiratory distress syndrome, lobar collapse, and a requirement for noninvasive or invasive ventilation..$^{5-7}$ Currently 11 to $31 \%$ of patients with MRFs will develop pneumonia, ${ }^{6,7}$ with odds of mortality nearly four times that of those without. ${ }^{8}$

Good analgesia allows these patients to cough, deep breathe, and participate in their physiotherapy sessions. These factors have been shown to reduce the risk of pneumonia and mortality. ${ }^{5,9}$ It also has a role in improving patient comfort and reduces the development of chronic pain. ${ }^{10,11}$ A multidisciplinary clinical pathway targeting pain-control services for these high-risk trauma patients decreased rib fracture-associated morbidity and mortality. ${ }^{12}$ Rib fracture scoring systems offer a unique clinical utility to identify high-risk patients and have been combined with pain pathways to ensure that the pain of high-risk patients is managed aggressively. ${ }^{11,13}$

Over the last decade, the specialist societies ${ }^{14}$ have downgraded the usage of epidural as the gold standard for MRF pain. Their advice was adjusted following further review of available literature after the exclusion of postoperative surgical patients. The overall consensus is that the current evidence base regarding the optimal
1,3,4,6 Department of Anesthesia and Critical Care, Queen Elizabeth Hospital Birmingham, Birmingham, UK

${ }^{2}$ Department of Anesthesia and Critical Care, Leeds Teaching Hospitals NHS Trust, Leeds, UK

${ }^{5}$ Birmingham Acute Care Research Group, University of Birmingham, Birmingham, UK

Corresponding Author: Laura Beard, Department of Anesthesia and Critical Care, Queen Elizabeth Hospital Birmingham, Birmingham, UK, Phone: +44 (0)7825778064, e-mail: laura.beard@nhs.net

How to cite this article: Beard L, Holt B, Snelson C, Parcha C, Smith FG, Veenith T. Analgesia of Patients with Multiple Rib Fractures in Critical Care: A Survey of Healthcare Professionals in the UK. Indian J Crit Care Med 2020;24(3):184-189.

Source of support: Fang Gao Smith is supported by the NIHR Senior Investigator Award. Tonny Veenith is supported by NIHR, Clinical Research Network and the NIAA. Laura Beard has had conference travel fees funded by Pajunk.

Conflict of interest: None

analgesic method in this cohort is limited and of low quality. ${ }^{14}$ Current regional techniques in use for patients with MRFs include TEA, paravertebral catheters (PVB), intrapleural infusion, and the more recent thoracic wall fascial plane techniques such as the serratus anterior plane (SAP) and erector spinae plane (ESPB) blocks. Patients with MRFs represent a large high-risk cohort in whom it is essential that pain is managed rapidly and aggressively. 
However, achieving adequate analgesia can be challenging due to the presence of associated thoracic spine injury, comorbidities, trauma-related coagulopathy and prior anticoagulant use. ${ }^{15}$

We designed this survey to explore the current analgesic practice in the UK, the use of analgesic protocols, barriers to analgesic provision, and physician analgesic preferences. The results of the survey would be used to help inform further research into the analgesic management of this cohort and the development of a standardized validated national analgesic protocol.

\section{Materials and Methods}

We distributed the survey nationally to an enriched cohort of clinicians working in trauma units with an interest in MRF management over a 6-week period in 2018 (April/May). To make the survey generalizable, we asked various members of the trauma clinical team, including intensivists, anesthetists, surgeons, and advanced critical care practitioners (ACCPs). No incentive was provided to encourage participation.

\section{Survey Design}

This survey was designed by members of the intensive care unit (ICU) using a web-based platform. Questions were selected to identify the respondents' demographics (role, specialty, and region), local practices of managing rib fracture-associated pain, their attitudes toward these practices, and any barriers they have experienced in providing analgesia for MRF patients. In order to prevent the formation of an excess of data categories during analysis, respondents were presented with a series of answers to select from which had been deemed relevant by practitioners in the design team. Respondents were also permitted to specify their own answers where appropriate as free text. Answer choices were determined based on the question posed. A range of discrete options were used to assess demographics and the method of analgesia most commonly employed, and a 5-point Likert scale (always, usually, sometimes, rarely, never) was used to assess the frequency of adherence to protocols where present. Statistical analysis involved a Chi-square test to determine the relationship between using an analgesic protocol and adequacy of analgesia. A $p$ value of $<0.05$ was considered significant.

\section{Results}

Seventy-nine healthcare professionals completed the survey. Nine of these surveys were incomplete and thus excluded.

\section{Demographics of Respondents}

The majority of respondents were physicians $(n=44)$ at varying stages of training: consultants, specialty trainees, foundation or core trainees, and nontraining doctors, accounting for 26, 15, 2, and 1 of the respondents, respectively. The remaining respondents consisted of 20 ACCPs, 5 nurses, and 1 unspecified staff role (Table 1). Of all the specialties surveyed, the respondents mostly worked in intensive care in some capacity $(n=46,65.7 \%)$, primarily mixed, followed by anesthesia ( $n=20,28.6 \%)$, surgery with an interest in trauma ( $n=3,4.3 \%)$, and emergency medicine ( $n=1,1.4 \%)$. The majority of respondents worked in a tertiary center with regional trauma facilities available also known as a major trauma center [MTC $(82.9 \% n=58)]$. In UK, the MTCs were introduced in 2012 to standardize and improve the immediate care and rehabilitation services for patients who experience life- or limb-threatening
Table 1: Demographics of survey respondents by staff role, specialty, area of interest in intensive care unit, and type of hospital

\begin{tabular}{|c|c|c|c|}
\hline & & $n$ & $\%$ \\
\hline \multirow[t]{7}{*}{ Staff role } & Consultant & 26 & 37.1 \\
\hline & Specialty trainee/registrar & 15 & 21.4 \\
\hline & Foundation/core trainee & 2 & 2.9 \\
\hline & Nontraining doctor & 1 & 1.4 \\
\hline & Nurse & 5 & 7.1 \\
\hline & $\mathrm{ACCP}$ & 20 & 28.6 \\
\hline & Other & 1 & 1.4 \\
\hline \multirow[t]{7}{*}{ Specialty } & Anesthetics & 20 & 28.6 \\
\hline & Emergency medicine & 1 & 1.4 \\
\hline & ICU & 26 & 37.1 \\
\hline & ICU and anesthetics & 16 & 22.9 \\
\hline & ICU and general medicine & 4 & 5.7 \\
\hline & Surgery (with trauma) & 3 & 4.3 \\
\hline & Surgery (without trauma) & 0 & 0.0 \\
\hline \multirow[t]{8}{*}{ Area of ICU } & General (surgery) & 3 & 4.3 \\
\hline & General (medical) & 0 & 0.0 \\
\hline & General (mixed) & 44 & 62.9 \\
\hline & Cardiothoracic & 6 & 8.6 \\
\hline & Liver & 4 & 5.7 \\
\hline & Neuro & 8 & 11.4 \\
\hline & Burns & 1 & 1.4 \\
\hline & Other & 4 & 5.7 \\
\hline \multirow[t]{6}{*}{$\begin{array}{l}\text { Type of } \\
\text { hospital }\end{array}$} & $\begin{array}{l}\text { Tertiary center with regional } \\
\text { trauma center }\end{array}$ & 58 & 82.9 \\
\hline & $\begin{array}{l}\text { Tertiary center without regional } \\
\text { trauma center }\end{array}$ & 2 & 2.9 \\
\hline & $\begin{array}{l}\text { Tertiary pediatric center with } \\
\text { trauma }\end{array}$ & 0 & 0.0 \\
\hline & $\begin{array}{l}\text { Tertiary pediatric center without } \\
\text { trauma }\end{array}$ & 0 & 0.0 \\
\hline & District general hospital & 9 & 12.9 \\
\hline & Single specialty hospital & 1 & 1.4 \\
\hline
\end{tabular}

traumatic injuries. The MTCs are usually located within larger hospitals in major cities and have immediate access to computed tomography scanners, operating theaters, and critical care services. Of the respondents, $92.3 \%$ were from England where the majority of MTCs are located, with the remainder located in Wales and Scotland.

Of the respondents, $31.4 \%$ reported that their department has a protocol in place for the analgesia of patients with rib fractures, $52.9 \%$ stated their department did not, and $15.7 \%$ were unsure (Fig. 1A). Of those that did have a protocol in their place of work, $27.3 \%$ reported they always used the protocol, $54.6 \%$ reported they usually used the protocol, $9.1 \%$ used it sometimes and $9.1 \%$ never (Fig. 1B).

In addition to paracetamol, nonsteroidal anti-inflammatory drugs (NSAIDs), and morphine, Patient-controlled analgesia (PCA) was the most commonly used form of analgesia for $38.6 \%$ of respondents, followed by TEA (30.0\%) and continuous infusion of drugs such as morphine, alfentanil, or ketamine (18.6\%) (Fig. 2A). Patient-controlled analgesia was also the second most commonly used form of analgesia, popular among those who do not use it first-line. 

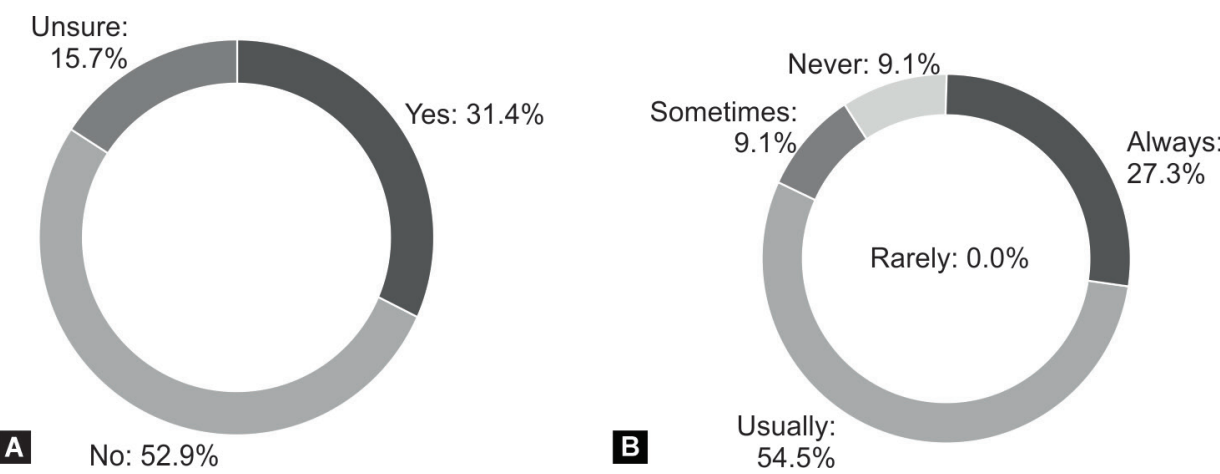

Figs $1 \mathrm{~A}$ and $\mathrm{B}$ : Access to and usage frequency of analgesia protocols for managing rib fracture-associated pain among respondents; (A) Accessibility; (B) Usage frequency among those with access

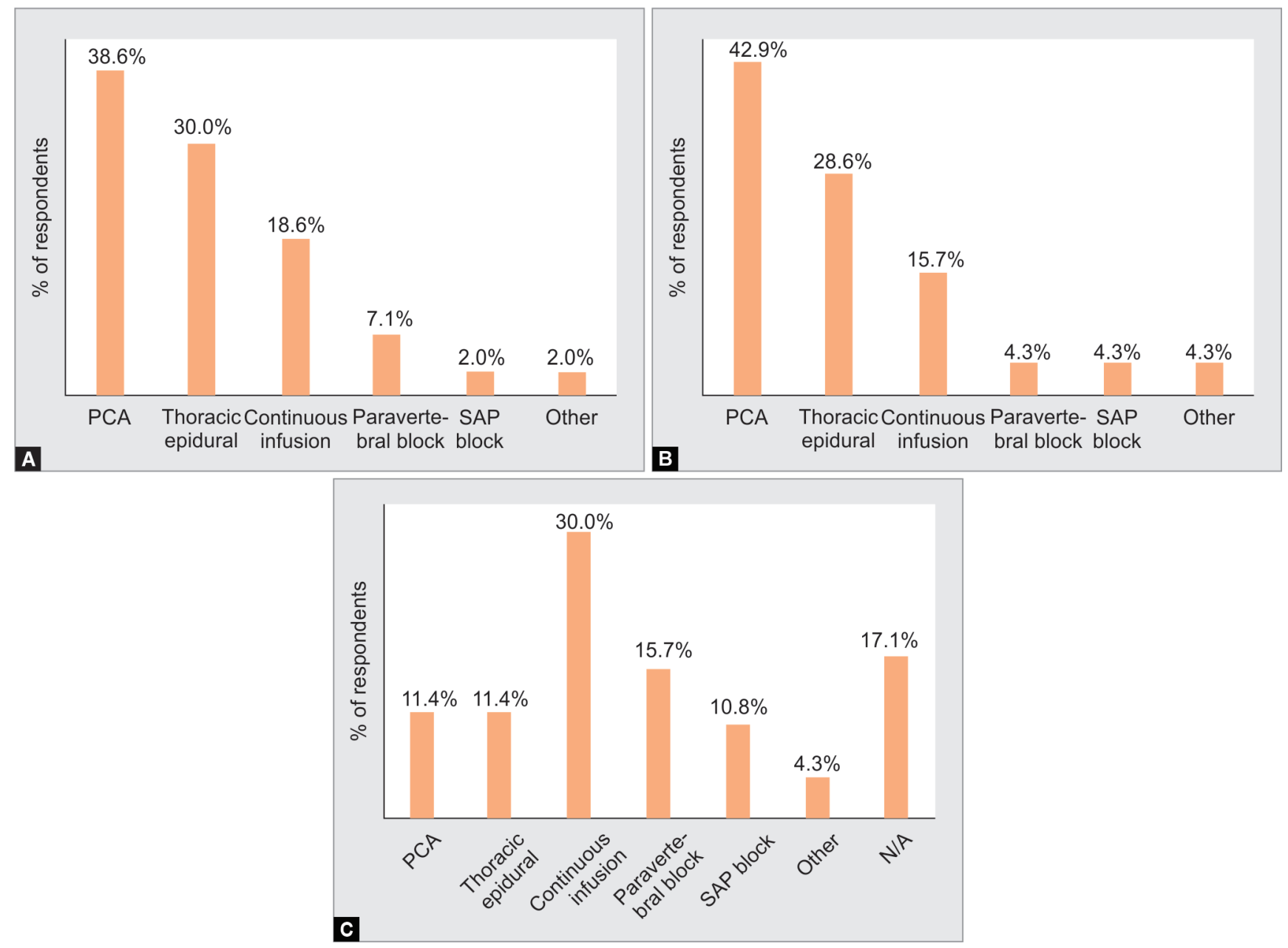

Figs 2A to C: Most frequently used methods of analgesia for rib fracture-associated pain in intensive care; (A) Most commonly used; (B) Second most commonly used; (C) Third most commonly used

Paravertebral catheters and SAP were not commonly used as first- or second-line methods, together accounting for $9.1 \%$ and $8.6 \%$ of these, respectively (Figs $2 \mathrm{~A}$ and B). Their usage collectively increased to $26.5 \%$ when the respondents considered the third most commonly used method (Fig. 2C).

In the presence of no contraindications, TEA was the preferred method of analgesia for rib fractures among respondents, which was opted for by $37.1 \%$ vs $14.3 \%$ for PCA. Paravertebral catheters and SAP were also more frequently used in the absence of contraindications, being the method of choice for $17.1 \%$ and $21.4 \%$ of respondents, respectively (Fig. 3).

\section{Attitudes Concerning the Efficacy of Analgesia}

When asked whether they thought the pain of patients with rib fractures was adequately managed in the ICU, no respondents reported that pain was "always" controlled; $42.9 \%$ reported it was 


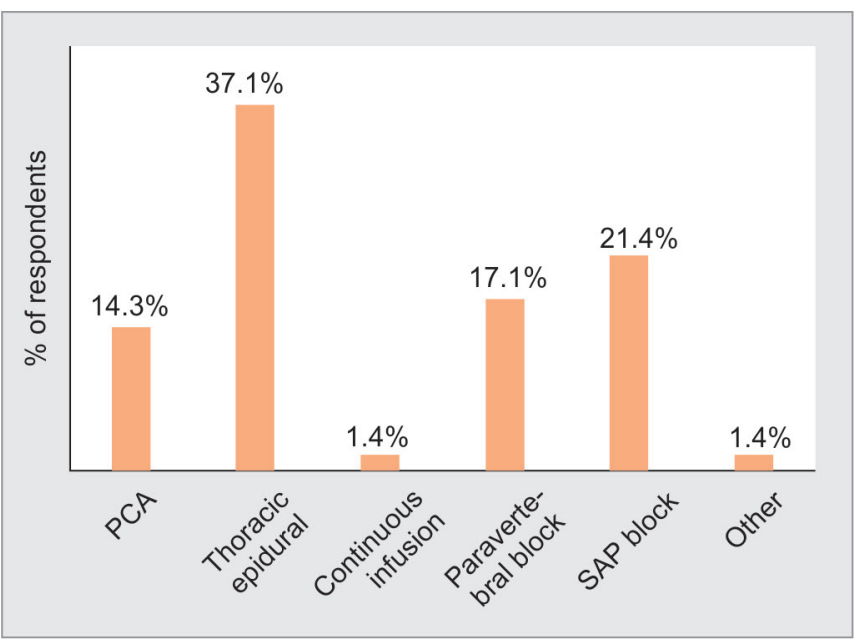

Fig. 3: The respondents' preferred method of choice for managing pain secondary to rib fractures in the absence of any contraindications

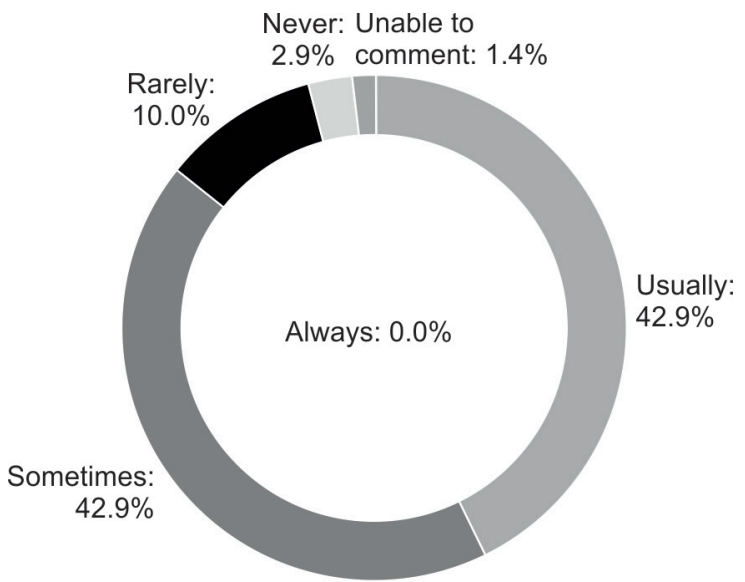

Fig. 4: Frequency of adequate analgesia being achieved for multiple rib fracture patients in intensive care

Table 2: Adequacy of pain control with and without the presence of pain management guidelines

\begin{tabular}{|c|c|c|c|c|c|c|c|}
\hline \multicolumn{8}{|c|}{ How often do you feel pain is adequately managed? } \\
\hline $\begin{array}{l}\text { Do you have a } \\
\text { guideline? }\end{array}$ & $\begin{array}{l}\text { Number of } \\
\text { responses }\end{array}$ & Always & Usually & Sometimes & Rarely & Never & $\begin{array}{l}\text { Unable to } \\
\text { comment }\end{array}$ \\
\hline Yes & 23 & 0 & 18 & 4 & 0 & 0 & 0 \\
\hline No & 41 & 0 & 8 & 23 & 6 & 1 & 3 \\
\hline
\end{tabular}

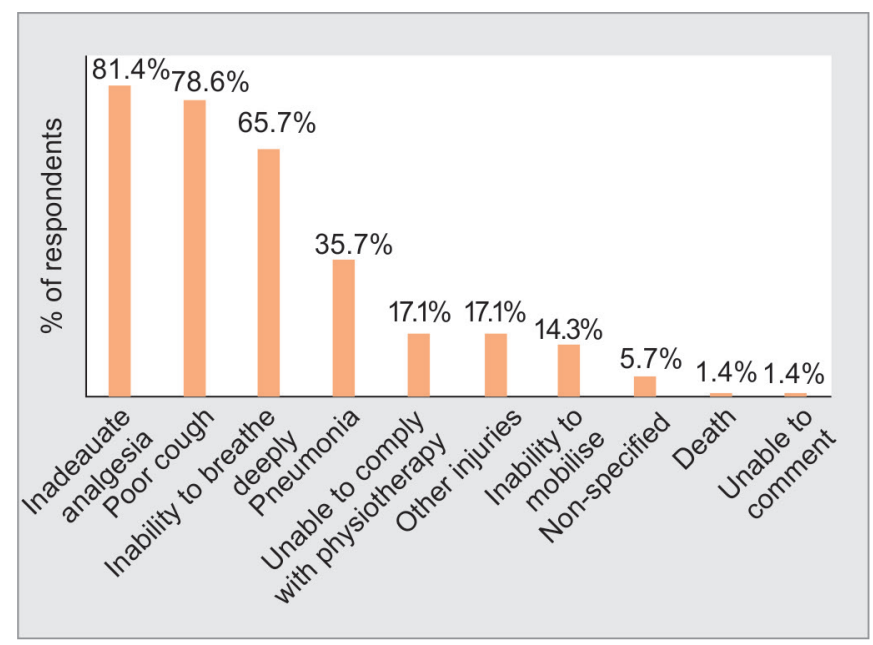

Fig. 5: Most frequent complications of rib fractures seen by respondents in intensive care (up to three complications selected per respondents)

"usually" controlled, 42.9\% "sometimes," 10.0\% "rarely," and 2.9\% "never"; $1.4 \%$ was unable to comment (Fig. 4).

A comparison of the adequacy of analgesia was made between those who responded that they had a protocol compared to those that did not (Table 2). Respondents who were uncertain about the presence of a hospital protocol were excluded. Adequate pain control was taken to be "always" and "usually" and inadequate pain control "sometimes," "rarely," and "never."

For those with a hospital guideline, adequate pain control was reported by $78.3 \%$ and inadequate by $17.4 \%$, compared to those without a hospital protocol in which adequate pain control was reported by $21.1 \%$ and inadequate control by $78.9 \%$.
Significantly more respondents who had a hospital protocol said they achieved adequate pain control compared to those without $\left(\chi^{2}, p<0.01\right)$.

Respondents were also asked which three complications of rib fractures they most often encountered in their ICU. These were found to be inadequate analgesia (81.4\%), a poor cough (78.6\%), and inability to breathe deeply (65.7\%); (Fig. 5).

\section{Barriers to Analgesia}

Nearly two thirds of respondents (60.0\%) reported challenges or barriers to analgesic options for MRF patients in the ICU. A fifth (21.4\%) experienced neither of these, while the remainder (18.6\%) were unable to comment (Fig. 6A). Each respondent selected up to five reasons for this. Contraindications provided the majority of challenges (69.0\%), closely followed by a lack of skilled staff (64.3\%), service and time pressures (52.4\%), colleague objection (50.0\%), and patient positioning (47.6\%) (Fig. 6B). These barriers were in respect to all methods of analgesia collectively.

\section{Discussion}

\section{Current Practice}

These results demonstrate that there is considerable variation among physicians in their current use of analgesic modalities for patients with MRFs. Opiate-based methods predominate, with PCAs and continuous infusions still the mainstay of analgesic treatment for these patients. This is supported by the current literature. A retrospective review of patients admitted with rib fractures recorded that $63 \%$ of patients received opiate-based PCA analgesia and $18 \%$ TEA. ${ }^{16}$ Other studies report TEA use between 9.9 and $18.4 \% .6,7,17$ In this study when no contraindications are present, physicians preferred method of analgesia was TEA, closely 


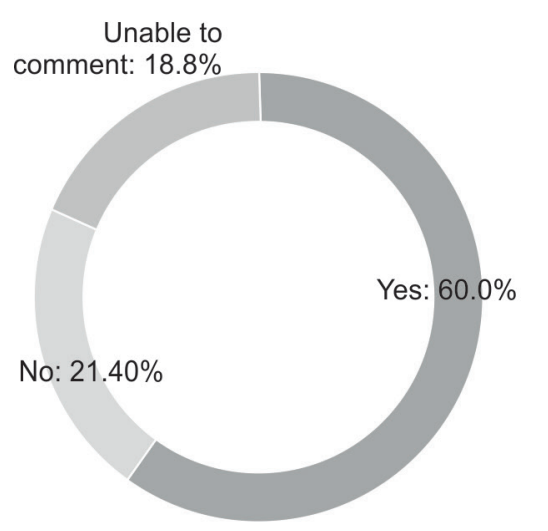

A

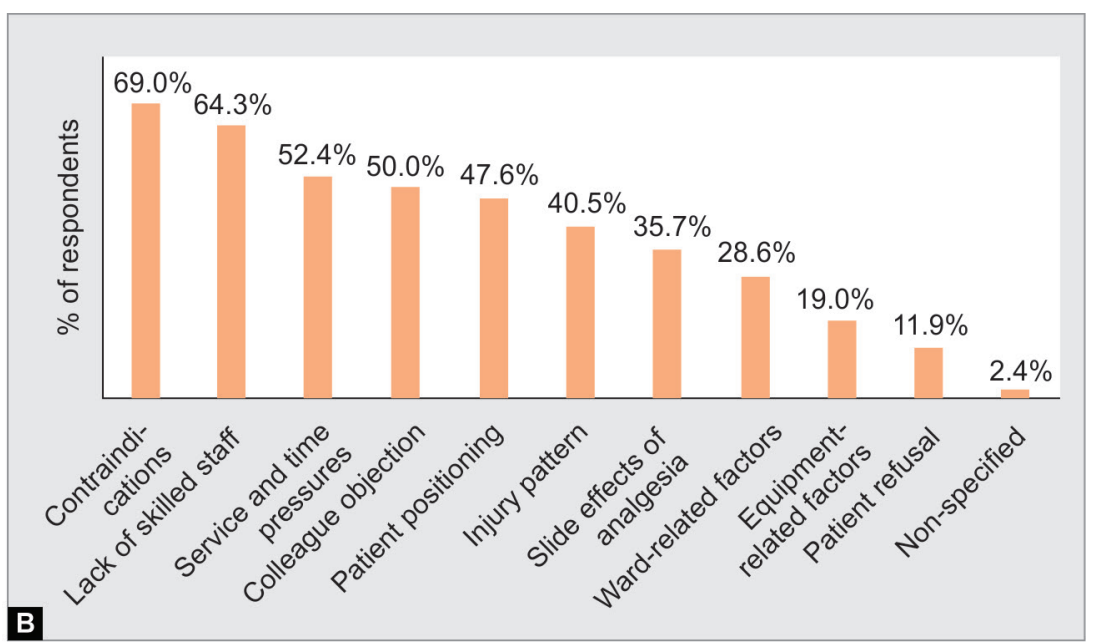

Figs $6 A$ and $B:(A)$ The percentages of respondents who have or have not experienced barriers to or challenges in providing analgesia for patients with rib fractures; (B) The most common barriers and challenges experienced by respondents in intensive care

followed by SAP and PVB (37.1\%, 21.4\%, and 17.1\%), thus revealing an overwhelming preference for regional anesthesia where possible.

This is perhaps understandable, given the benefits of regional anesthesia in other cohorts. These advantages include superior analgesia and the avoidance of adverse effects associated with opioid use such as nausea and vomiting, constipation, sedation, and delirium. ${ }^{18}$

\section{Barriers to Analgesia}

This study found that barriers to the provision of analgesia were experienced by $60.0 \%$ of respondents. Contraindications to analgesic choice, lack of skilled staff and service and time pressures were the top three barriers to providing analgesia (69.0\%, $64.3 \%$, and $52.4 \%)$. Thoracic epidural use can be problematic in this cohort as up to $90 \%$ of patients with MRFs have associated injuries such as spinal and head injuries which, in addition to preexisting comorbidities, hypotension, and coagulopathy, may preclude their use. ${ }^{6}$ This might also explain why TEA usage in the literature and this study is limited despite being the preferred choice by physicians. Opioid-based analgesia, though less favored by physicians in this study, is the most commonly used analgesic method. This is due to its noninvasive nature, ease of administration, and ability for it to be provided by a range of healthcare professionals.

Alternative techniques such as the novel thoracic wall blocks will potentially play a larger role in the management of pain secondary to MRFs. Serratus anterior plane and ESPB are less susceptible to the barriers mentioned above. Their insertion site is away from the epidural space, increasing the access of patients with MRFs to regional techniques due to a reduced contraindication profile. There is also the potential for nonanesthetists to perform the SAP catheter insertion because of its superficial insertion site and reduced complication risk compared to PVB and TEA. ${ }^{19}$ Serratus anterior plane can also be performed in the supine position, negating the need for patient repositioning. These factors would be significant where time and staff skill mix are the limitations. Barriers aside, the regional technique that is superior for managing rib fracture pain is yet to be determined. ${ }^{14}$ Thoracic epidural has long been considered the gold standard of thoracic analgesia but associated adverse effects and complications detract from its merits.

A recent study demonstrated SAP provides an equivalent level of analgesia to TEA postthoracotomy while maintaining hemodynamic stability. ${ }^{20}$ Similarly, a meta-analysis of PVB vs TEA postthoracotomy found no significant difference in reported pain scores. ${ }^{21}$ Evidence suggests PVB may provide superior analgesia to SAP. A recent trial comparing these two regional blocks following breast surgery observed a prolonged duration of analgesia and reduced opioid usage associated with PVB. ${ }^{22}$ However, PVB is a more difficult technique to perform with the potential for greater complications. ${ }^{11,23}$ Further high-quality studies are required to fully investigate the efficacy of the novel SAP and ESPB techniques compared to the more traditional regional approaches of TEA and PVB in the trauma cohort.

\section{Guideline Adoption}

It is concerning that no respondents felt that patient's pain was "always" controlled. Inadequate pain control was the most common complication reported (81.4\%), closely followed by poor cough (78.6\%) and inability to deep breathe (65.7\%), both exacerbated by inadequate analgesia. Previous studies demonstrated the effectiveness of multidisciplinary patient pathways to improve outcomes for patients with MRFs. ${ }^{12}$ Witt and Bulger also showed the benefits of clinical scoring systems and early multimodal analgesia. ${ }^{4}$

The use of guidelines and protocols with associated severity scoring systems to assist with the escalation of pain management is essential. Approximately one third (31.4\%) of respondents had a protocol for analgesia in their place of work, with $81.8 \%$ of respondents stating they always or usually followed the protocol, suggesting that protocols are well utilized when present.

This study revealed the pain relief of patients with MRFs was perceived to be significantly better managed when a hospital protocol was present compared to not $\left(\chi^{2}, p<0.01\right)$.

\section{Conclusion}

These results show that the current analgesic practice for patients with MRFs is inadequate. Uptake of protocols that provide escalation strategies ${ }^{11}$ is not universally adopted. However, protocol use in this cohort is associated with improved analgesia and patient outcomes. Regional anesthetic techniques such as TEA are preferred over opiates by physicians but opiate use predominates in those with thoracic injuries as a result of contraindications to TEA use, 
service and time pressures, and staff skill mix. The novel thoracic wall blocks with their reduced side effect profile, ease of insertion, and limited contraindications may herald a new approach to the analgesic management of patients with MRFs. Further research into the efficacy of these thoracic wall blocks is needed.

Although it is recognized that regional analgesia is very important for the pain management of patients with thoracic trauma, there is still an important role for PCA morphine, especially in those with polytrauma and also to allow the patient autonomy over their analgesic regime. In patients with multiple injuries, morphine PCA and regional analgesia should be seen as complementary techniques aiming to improve pain, patient satisfaction, and reduce opiate consumption.

We suggest future research should focus on currently utilized regional techniques of TEA, PVB, SAP, and ESPB. In the UK, the use of pleural infusions and intercostal nerve catheters as mentioned in some specialty guidance is a rarity. ${ }^{14}$ The aim of such research would be to produce a validated standardized national protocol that is informed by current practice, the evidence base, and current limitations to service provision.

\section{References}

1. Pressley CM, Fry WR, Philp AS, Berry SD, Smith RS. Predicting outcome of patients with chest wall injury. Am J Surg 2012;204(6):910-913; discussion 3-4 10.1016/j.amjsurg.2012.05.015.

2. Lin FC, Li RY, Tung YW, Jeng KC, Tsai SC. Morbidity, mortality, associated injuries, and management of traumatic rib fractures. J Chin Med Assoc 2016;79(6):329-334. DOI: 10.1016/j.jcma.2016.01.006.

3. Liman S, Kuzucu A, Tastepe A, Ulsan G, Topcu S. Chest injury due to blunt trauma. Eur J Cardiothorac Surg 2003;23(3):374-378. DOI: 10.1016/s1010-7940(02)00813-8.

4. Witt CE, Bulger EM. Comprehensive approach to the management of the patient with multiple rib fractures: a review and introduction of a bundled rib fracture management protocol. Trauma Surg Acute Care Open 2017;2(1):e000064. DOI: 10.1136/tsaco-2016-000064.

5. Bulger EM, Edwards T, KlotzP, Jurkovich GJ. Epidural analgesia improves outcome after multiple rib fractures. Surgery 2004;136(2):426-430. DOI: 10.1016/j.surg.2004.05.019.

6. Chapman BC, Herbert B, Rodil M, Salotto J, Stovall RT, Biffl W, et al. RibScore: a novel radiographic score based on fracture pattern that predicts pneumonia, respiratory failure, and tracheostomy. J Trauma Acute Care Surg 2016;80(1):95-101. DOI: 10.1097/ TA.0000000000000867.

7. Jensen CD, Stark JT, Jacobson LL, Powers JM, Joseph MF, KinsellaShaw JM, et al. Improved outcomes associated with the liberal use of thoracic epidural analgesia in patients with rib fractures. Pain Med 2017;18(9):1787-1794. DOI: 10.1093/pm/pnw199.

8. Battle $\mathrm{CE}$, Hutchings $\mathrm{H}$, Evans PA. Risk factors that predict mortality in patients with blunt chest wall trauma: a systematic review and metaanalysis. Injury 2012;43(1):8-17. DOI: 10.1016/j.injury.2011.01.004.

9. Bulger EM, Arneson MA, Mock CN, Jurkovich GJ. Rib fractures in the elderly. J Trauma 2000;48(6):1040-1047. DOI: 10.1097/00005373200006000-00007.
10. Hasenboehler EA, Bernard AC, Bottiggi AJ, Moghadamian ES, Wright RD, Chang PK, et al. Treatment of traumatic flail chest with muscular sparing open reduction and internal fixation: description of a surgical technique. J Trauma 2011;71(2):494-501. DOI: 10.1097/ TA.0b013e3182255d30.

11. May L, Hillermann C, Patil S. Rib fracture management. BJA Educ 2016;16(1):26-32. DOI: 10.1093/bjaceaccp/mkv011.

12. Todd SR, McNally MM, Holcomb JB, Kozar RA, Kao LS, Gonzalez $E A$, et al. A multidisciplinary clinical pathway decreases rib fracture-associated infectious morbidity and mortality in highrisk trauma patients. Am J Surg 2006;192(6):806-811. DOI: 10.1016/ j.amjsurg.2006.08.048.

13. Maxwell CA, Mion LC, Dietrich MS. Hospitalized injured older adults: clinical utility of a rib fracture scoring system. J Trauma Nurs 2012;19(3):168-174. ; quiz 75-76 10.1097/JTN.0b013e318261d201.

14. Galvagno Jr SM, Smith CE, Varon AJ, Hasenboehler EA, Sultan S, Shaefer $G$, et al. Pain management for blunt thoracic trauma: a joint practice management guideline from the Eastern Association for the Surgery of Trauma and Trauma Anesthesiology Society. J Trauma Acute Care Surg 2016;81(5):936-951. DOI: 10.1097/ TA.0000000000001209.

15. Bulger EM, Edwards WT, de Pinto M, Klotz P, Jurkovich GJ. Indications and contraindications for thoracic epidural analgesia in multiply injured patients. Acute Pain 2008;10(1):15-22. DOI: 10.1016/ j.acpain.2007.10.019.

16. Chincholkar M, Mills J, Howarth M, Murison F, Dwyer A, Makin R. Winning abstracts from the National Acute Pain Symposium 2017. Pain management in trauma associated with rib fractures. Br J Pain 2017;11(4):210-212. DOI: 10.1177/2049463717736491

17. Carver TW, Milia DJ, Somberg C, Brasel K, Paul J. Vital capacity helps predict pulmonary complications after rib fractures. J Trauma Acute Care Surg 2015;79(3):413-416. DOI: 10.1097/TA.0000000000000744.

18. Choi J, Gadsden J. Chapter 10: local anaesthetic mixtures for peripheral nerve blocks. In: Hadzic A, ed. Hadzic's Textbook of Regional Anesthesia and Acute Pain Management. 2nd ed., New York: McGraw-Hill; 2017.

19. Durant E, Dixon B, Luftig J, Mantuani D, Herring A. Ultrasound-guided serratus plane block for ED rib fracture pain control. Am J Emerg Med 2017;35(1):197.e3-197.e6. DOI: 10.1016/j.ajem.2016.07.021.

20. Khalil AE, Abdallah NM, Bashandy GM, Kaddah TA. Ultrasoundguided serratus anterior plane block vs thoracic epidural analgesia for thoracotomy pain. J Cardiothorac Vasc Anesth 2017;31(1):152-158. DOI: 10.1053/j.jvca.2016.08.023.

21. Davies RG, Myles PS, Graham JM. A comparison of the analgesic efficacy and side-effects of paravertebral vs epidural blockade for thoracotomy--a systematic review and meta-analysis of randomized trials. Br J Anaesth 2006;96(4):418-426. DOI: 10.1093/bja/ ael020.

22. Gupta K, Srikanth K, Girdhar KK, Chan V. Analgesic efficacy of ultrasound-guided paravertebral block vs serratus plane block for modified radical mastectomy: a randomised, controlled trial. Indian J Anaesth 2017;61(5):381-386. DOI: 10.4103/ija.IJA_ 62_17.

23. Kaur RP, Anwar S. Serratus anterior plane block vs thoracic epidural analgesia for post-thoracotomy pain relief. J Cardiothorac Vasc Anesth 2018;32(6):11-12. DOI: 10.1053/j.jvca.2018.05.046. 